\title{
MODULI SPACE OF BRANCHED SUPERMINIMAL IMMERSIONS OF A COMPACT RIEMANN SURFACE INTO $\mathrm{S}^{4}$
}

\author{
BONAVENTURE LOO
}

(Received 15 March 1997; revised 1 September 1998)

Communicated by P. C. Fenton

\begin{abstract}
In this paper we describe the moduli spaces of degree $d$ branched superminimal immersions of a compact Riemann surface of genus $g$ into $S^{4}$. We prove that when $d \geq \max \{2 g, g+2\}$, such spaces have the structure of projectivized fibre products and are path-connected quasi-projective varieties of dimension $2 d-g+4$. This generalizes known results for spaces of harmonic 2 -spheres in $S^{4}$.

1991 Mathematics subject classification (Amer. Math. Soc.): primary 58D27; secondary 58E20, 53A10, $53 \mathrm{C} 15,32 \mathrm{~L} 25$.
\end{abstract}

In the Calabi construction (see [C]) the space of harmonic maps (or, equivalently, the space of branched minimal immersions) of $S^{2}$ into $S^{2 n}$ decomposes into a union of moduli spaces $H_{k}$ labelled by the harmonic degree $k \geq 0$. (This means that if $f \in H_{k}$, then $\operatorname{Area}\left(f\left(S^{2}\right)\right)=\operatorname{Energy}(f)=4 \pi k$.) When $f$ is linearly full (that is, its image is not contained in a totally geodesic subsphere), then either $f$ or $-f$ (that is, $f$ followed by the antipodal map on $S^{2 n}$ ) has a holomorphic horizontal lift to the twistor space of $S^{2 n}$ such that its image is a curve of degree $k$. In the case when $n=2$, if $f$ is totally geodesic, then both $f$ and $-f$ have holomorphic horizontal lifts to the twistor space $\mathbb{C} \mathbb{P}^{3}$, and their images in $\mathbb{C} \mathbb{P}^{3}$ are linear projective lines. The study of the space of harmonic maps of $S^{2}$ into $S^{4}$ (modulo the antipodal map on $S^{4}$ ) thus reduces to the study of holomorphic horizontal curves (of genus 0 ) in $\mathbb{C P}^{3}$. In [L] the study of holomorphic horizontal curves of degree $k$ and genus 0 in $\mathbb{C P}^{3}$ was reduced to the study of the moduli space $\mathscr{M}_{k}$ of pairs of meromorphic functions of degree $k$ with the same ramification divisor.

Recall from Wirtinger's inequality that a holomorphic curve in a Kähler manifold is area minimizing in its homology class. Since the twistor projection $\mathbb{C P}^{3} \rightarrow S^{4}$ is a

(C) 1999 Australian Mathematical Society 0263-6115/99 $\$ A 2.00+0.00$ 
Riemannian submersion, the projection of a holomorphic horizontal curve in $\mathbb{C} \mathbb{P}^{3}$ is automatically minimal in $S^{4}$. By general twistor theory, such maps are also harmonic (see [BR] and [ES] for example). For a fixed compact Riemann surface $\Sigma$ of genus $g>0$, we shall consider the special class of harmonic maps from $\Sigma$ to $S^{4}$ which admit holomorphic horizontal liftings to $\mathbb{C P}^{3}$. Such maps are called 'branched superminimal immersions'. In 1982 Robert Bryant obtained the following result:

THEOREM ([Br]]). Every compact Riemann surface admits a superminimal immersion into $S^{4}$.

In this paper, we shall study the moduli spaces of all such maps. We shall give a direct construction for holomorphic horizontal curves of genus $g$. Since every such curve in $\mathbb{C P}^{3}$ has a degree, we can classify the moduli spaces by the degree of the curves. For a fixed compact Riemann surface $\Sigma$ of genus $g$, and a fixed degree $d$, we shall let $\mathscr{S}_{d . \Sigma}$ denote the moduli space of branched superminimal immersions of $\Sigma$ into $S^{4}$ of degree $d$ (equivalently, the moduli space of degree $d$ holomorphic horizontal maps of $\Sigma$ to $\mathbb{C P}^{3}$ ). We shall abuse language by using the terms 'curve in $\mathbb{C P}^{3}$ ' and 'holomorphic map of $\Sigma$ to $\mathbb{C P}^{3}$ ' interchangeably.

In the first section, we recall the basic construction given in [L]. The second section is concerned with using fibre products to describe the moduli space. This is done by first fixing a line bundle of degree $d$ over a fixed Riemann surface $\Sigma$ of genus $g$. In Section 3, we let the line bundle vary over the Jacobian variety of degree $d$ line bundles over $\Sigma$ and we obtain results on the dimension of the moduli space. Pathconnectedness of the moduli space is discussed in Section 4. We obtain the following main result.

THEOREM 4.1. For $d \geq \max \{2 g, g+2\}, \mathscr{S}_{d . \Sigma}$ is a path-connected quasi-projective variety of dimension $2 d-g+4$.

In Section 5 we discuss compactifications of the moduli space. We provide some simple examples of elements in the boundary of the moduli space.

\section{Superminimal immersions and contact curves}

Let us first recall the twistor fibration $\mathbb{C P}^{1} \rightarrow \mathbb{C} \mathbb{P}^{3} \rightarrow S^{4}$. Observe that $\mathbb{H}^{2}$ has the structure of a quaternion module with right scalar multiplication. By taking the quotient of $\mathbb{H}^{2} \backslash\{0\}$ by the action of right scalar multiplication by nonzero quaternions we obtain $\mathbb{H}^{\mathbb{1}}$. The natural identification of $\mathbb{C}^{4}$ with $\mathbb{H}^{2}$ via $\left(z_{0}, z_{1}, z_{2}, z_{3}\right) \mapsto\left(z_{0}+\right.$ $j z_{1}, z_{2}+j z_{3}$ ) gives us the formula for the twistor fibration over $S^{4} \cong \mathbb{H} \mathbb{P}^{\prime}$ :

$$
\mathbb{C P}^{3} \ni\left[z_{0}, z_{1}, z_{2}, z_{3}\right] \mapsto\left[z_{0}+j z_{1}, z_{2}+j z_{3}\right] \in \mathbb{H} \mathbb{P}^{1} .
$$


The Fubini-Study metric on $\mathbb{C P}^{3}$ splits the tangent space $T \mathbb{C P}^{3}$ into vertical and horizontal components. The horizontal distribution $\mathscr{H}$ has complex codimension 1 and is a holomorphic sub-bundle of $T \mathbb{C P}^{3}$. This distribution is a holomorphic contact structure-it is the kernel of a contact 1 -form $\theta$ on $\mathbb{C P}^{3}$ with values in a holomorphic line bundle $\mathscr{L}$, and it is encoded in the following exact sequence:

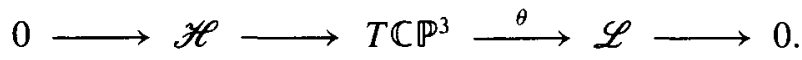

This contact form has a lifting to $\mathbb{C}^{4}$ given by

$$
\Omega=z_{0} d z_{1}-z_{1} d z_{0}+z_{2} d z_{3}-z_{3} d z_{2} .
$$

Since holomorphic horizontal curves are tangent to the distribution $\mathscr{H}$, we shall use the terms 'holomorphic horizontal curves' and 'contact curves' interchangeably.

We shall consider surfaces which arise as twistor projections of holomorphic curves tangent to the horizontal distribution in $\mathbb{C P}^{3}$. Such maps are known to be harmonic, and in fact branched minimal (see [Br], [La], [L], [ES] and [BR]).

DEFINITION 1.1. A harmonic map of a Riemann surface to $S^{4}$ is said to be branched superminimal if it is a projection of a horizontal holomorphic map into $\mathbb{C P}^{3}$.

In [L], such (generic) maps, modulo the 'contact involution' on $\mathbb{C P}^{3}$, were described in terms of pairs of meromorphic functions which satisfy a ramification condition. We summarize the construction of [L] below.

Consider the following two distinguished projective lines in $\mathbb{C P}^{3}$ :

$$
L_{0}:=\left\{\left[z_{0}, z_{1}, 0,0\right]\right\}, \quad L_{1}:=\left\{\left[0,0, z_{2}, z_{3}\right]\right\} .
$$

(These two distinguished lines project to the distinguished pair of antipodal points in $S^{4}$-the north and south poles.) We have a projection map

$$
\psi_{0}=\left(\pi_{0}, \pi_{1}\right): \mathbb{C P}^{3} \backslash\left(L_{0} \cup L_{1}\right) \longrightarrow L_{0} \times L_{1} \cong \mathbb{C P}^{1} \times \mathbb{C P}^{1},
$$

where $\pi_{0}$ and $\pi_{1}$ are respectively given by $x \mapsto \operatorname{span}\left(x, L_{1}\right) \cap L_{0}$ and $x \mapsto \operatorname{span}(x$, $\left.L_{0}\right) \cap L_{1}$. It was proved in [L] that $\psi_{0}$ has contact fibres. Letting $\widehat{C P P}^{3}$ denote the blow-up of $\mathbb{C} \mathbb{P}^{3}$ along $L_{0} \cup L_{1}$, we obtain a well-defined map

$$
\psi: \widehat{\mathbb{C P}}^{3} \longrightarrow \mathbb{C P}^{1} \times \mathbb{C P}^{1} .
$$

The map $\psi$ has contact fibres if we equip $\widehat{\mathbb{C P}}^{3}$ with the pull-back of the contact structure on $\mathbb{C P}^{3}$. We obtain the following diagram:

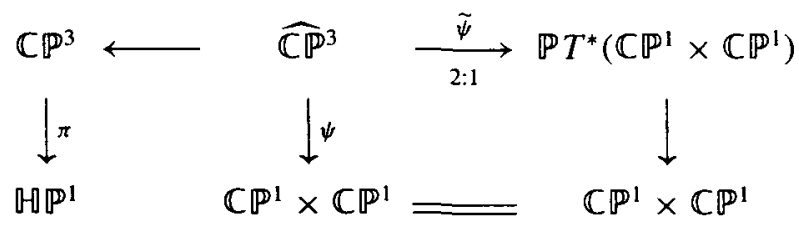


where $\tilde{\psi}$ is a contact $2: 1$ branched covering [L].

Observe that a generic holomorphic curve $\gamma \subset \mathbb{C} \mathbb{P}^{3}$ will miss a pair of skew lines, in particular, $\gamma \cap\left(L_{0} \cup L_{1}\right)=\emptyset$. (This implies that its image in $S^{4}$ lies in the complement of the two poles.) Let $\gamma$ be a generic holomorphic curve of degree $d$ in $\mathbb{C P}^{3}$. We obtain 2 meromorphic functions associated to the curve $\gamma$ :

$$
f_{i}:=\left.\pi_{i}\right|_{\gamma}: \gamma \longrightarrow L_{i}, \quad i=0,1,
$$

with

$$
\begin{aligned}
& \operatorname{deg} f_{0}=\#\left[\left(\operatorname{span}\left(x, L_{1}\right) \backslash L_{1}\right) \cap \gamma\right]=\#\left(\operatorname{span}\left(x, L_{1}\right) \cap \gamma\right)=\operatorname{deg}(\gamma)=d, \\
& \operatorname{deg} f_{1}=\#\left[\left(\operatorname{span}\left(y, L_{0}\right) \backslash L_{0}\right) \cap \gamma\right]=\#\left(\operatorname{span}\left(y, L_{0}\right) \cap \gamma\right)=\operatorname{deg}(\gamma)=d,
\end{aligned}
$$

where $x$ is any point in $L_{0}$ and $y$ is any point in $L_{1}$. Thus, a generic holomorphic curve of degree $d$ in $\mathbb{C} \mathbb{P}^{3}$ furnishes us with two meromorphic functions of degree $d$.

Let us examine a nongeneric case where $\gamma$ intersects one of the lines, say $L_{1}$, in $k$ points (counting multiplicity). Let $\tilde{\gamma}$ denote the proper transform of $\gamma$. We let $\pi_{i}$ denote the $i$-th factor projection map of (1.2) and let $f_{i}:=\left.\pi_{i}\right|_{\tilde{\gamma}}$. Then,

$$
\begin{aligned}
\operatorname{deg} f_{0} & =\#\left[\left(\operatorname{span}\left(x, L_{1}\right) \backslash L_{1}\right) \cap \gamma\right] \\
& =\#\left(\operatorname{span}\left(x, L_{1}\right) \cap \gamma\right)-\#\left(\gamma \cap L_{1}\right) \\
& =\operatorname{deg}(\gamma)-k=d-k,
\end{aligned}
$$

where $x$ is any point in $L_{0}$.

If in addition $\gamma: \Sigma \rightarrow \mathbb{C P}^{3}$ is a contact curve, then its proper transform $\tilde{\gamma}$ is a contact curve in $\widehat{C P}^{3}$ and we hence obtain a map $\Sigma \rightarrow \mathbb{C P}^{1} \times \mathbb{C} \mathbb{P}^{1}$ (that is, two meromorphic functions on $\Sigma$ ) which has a contact lift to $\mathbb{P} T^{*}\left(\mathbb{C P}^{1} \times \mathbb{C} \mathbb{P}^{1}\right)$ by (1.3). The contact condition on the curve implies that the two meromorphic functions have the same ramification divisor.

Note that any nonconstant holomorphic map $\sigma: \Sigma \rightarrow \mathbb{C P}^{1} \times \mathbb{C P}^{1}$ has a unique contact lift to $\mathbb{P} T^{*}\left(\mathbb{C} \mathbb{P}^{1} \times \mathbb{C P}^{1}\right)$ given by the Gauss lift $G(\sigma)=\left[\sigma \wedge \sigma^{\prime}\right]$. A lift of $G(\sigma)$ to $\widehat{C P}^{3}$ is a contact curve whose image in $\mathbb{C P}^{3}$ is nongeneric in the sense that it intersects the two distinguished lines. However, if $\sigma=\left(g_{0}, g_{1}\right)$ is of bidegree $(d, d)$ where $g_{0}$ and $g_{1}$ have the same ramification divisor, then $G(\sigma)$ lifts to a contact curve of degree $d$ in $\mathbb{C P}^{3}$ which avoids the two distinguished lines. This enables us to describe the moduli space of generic branched superminimal surfaces in $S^{4}$ of area $4 \pi d$ in terms of pairs of meromorphic functions with the same ramification divisor. Given such a pair of meromorphic functions, the construction given in [L] does not give an explicit description of the contact curve in $\mathbb{C P}^{3}$ - it only associates to such a pair of meromorphic functions a pair of contact curves via the map $\widetilde{\psi}$ in (1.3). In the next section, we shall show how one may obtain an explicit description given such holomorphic data. 


\section{Fibre products, Gaussian maps and Grassmannians}

Henceforth, we shall let $\Sigma$ be a fixed compact Riemann surface of genus $g$. Let $\gamma$ : $\Sigma \rightarrow \mathbb{C} \mathbb{P}^{3}$ be a holomorphic curve of degree $d$. Then there is a line bundle $L \rightarrow \Sigma$ of degree $d$ such that $\gamma(z)=\left[s_{0}(z), s_{1}(z), s_{2}(z), s_{3}(z)\right]$, where $s_{0}, s_{1}, s_{2}, s_{3} \in H^{0}(\Sigma, L)$. Note that the linear system $\left\langle s_{0}, s_{1}, s_{2}, s_{3}\right\rangle$ has no base points. If the pencils $\left\langle s_{0}, s_{1}\right\rangle$ and $\left\langle s_{2}, s_{3}\right\rangle$ are base-point-free, then the two meromorphic functions (thought of as holomorphic maps to $\mathbb{C P}^{1}$ ) arising from $\gamma$ are given by

$$
f_{0}=\left[s_{0}, s_{1}\right], \quad f_{1}=\left[s_{2}, s_{3}\right] .
$$

It follows from (1.1) that $\gamma$ is a contact curve if

$$
s_{0} s_{1}^{\prime}-s_{1} s_{0}^{\prime}+s_{2} s_{3}^{\prime}-s_{3} s_{2}^{\prime}=0 .
$$

For the rest of this section let $L \rightarrow \Sigma$ be a fixed line bundle of degree $d$. Let $W_{L}=H^{0}(\Sigma, L)$ and let $W_{L}^{k}$ denote the $k$-fold product of $W_{L}$. We wish to find four holomorphic sections of $L: s_{0}, s_{1}, s_{2}, s_{3} \in W_{L}$, such that (2.1) holds and where $\left\langle s_{0}, s_{1}, s_{2}, s_{3}\right\rangle$ has no base points. Consider the map

$$
\text { Ram : } \quad W_{L}^{2} \longrightarrow H^{0}\left(\Sigma, K L^{2}\right) \text { given by }(s, t) \longmapsto s d t-t d s,
$$

where $K$ is the canonical line bundle over $\Sigma$.

REMARK. Observe that $\operatorname{Ram}(s, t)=-\operatorname{Ram}(t, s)$. Thus, Ram is well defined on simple bivectors: $s \wedge t \mapsto s d t-t d s$. This extends linearly to a map

$$
\begin{aligned}
\Lambda: \bigwedge^{2} W_{L} & \longrightarrow H^{0}\left(\Sigma, K L^{2}\right) \quad \text { defined by } \\
\sum_{i<j} a_{i j} s_{i} \wedge s_{j} & \longmapsto \sum_{i<j} a_{i j}\left\{s_{i} d s_{j}-s_{j} d s_{i}\right\} .
\end{aligned}
$$

This map is well-known and it is called the Gaussian map ([W1], [W2]). It is called a Wahl map when $L=K$.

We can rewrite $(2.1)$ as $\operatorname{Ram}\left(s_{0}, s_{1}\right)+\operatorname{Ram}\left(s_{2}, s_{3}\right)=0$, that is,

$$
\operatorname{Ram}\left(s_{0}, s_{1}\right)=-\operatorname{Ram}\left(s_{2}, s_{3}\right) .
$$

In the case where the two pencils described above are base-point-free, this implies that the corresponding two functions, $f_{0}$ and $f_{1}$, have the same ramification divisor. Note that the ramification divisor of the function $f_{0}=\left[s_{0}, s_{1}\right]$ is just the divisor $\left(s_{0} d s_{1}-s_{1} d s_{0}\right) \in \mathbb{P} H^{0}\left(\Sigma, K L^{2}\right)$. 
Given a diagram

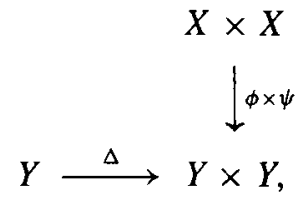

where $\Delta$ is the diagonal inclusion, recall that the fibre product $X \times_{Y} X$ is just the restriction to the diagonal of the product $\phi \times \psi: X \times X \rightarrow Y \times Y$, that is, $X \times_{Y} X=$ $\Delta^{*}(X \times X)$. From the diagram:

$$
\begin{aligned}
W_{L}^{2} \times W_{L}^{2}=W_{L}^{4} & \\
& \lfloor\operatorname{Ram} \times\{-\operatorname{Ram}\} \\
H^{0}\left(\Sigma, K L^{2}\right) \stackrel{\Delta}{\longrightarrow} H^{0}\left(\Sigma, K L^{2}\right) & \times H^{0}\left(\Sigma, K L^{2}\right),
\end{aligned}
$$

where $\Delta$ is the diagonal inclusion, consider the projectivized fibre product

$$
\mathscr{M}_{L, d}:=\mathbb{P}\left(W_{L}^{2} \times_{H^{0}\left(\Sigma, K L^{2}\right)} W_{L}^{2}\right) \subset \mathbb{P}\left(W_{L}^{4}\right) .
$$

REMARK. We adopt the following notation in this paper: given a vector space $V$, we let $\mathbb{P} V$ denote the projective space of lines through the origin in $V$.

Let $\mathscr{C}_{L, d}:=\left\{\left[s_{0}, s_{1}, s_{2}, s_{3}\right] \in \mathbb{P}\left(W_{L}^{4}\right) \mid\left\langle s_{0}, s_{1}, s_{2}, s_{3}\right\rangle\right.$ has base-points $\}$. Observe that each element of $\mathscr{C}_{L . d}$ corresponds to a parametrized curve in $\mathbb{C} \mathbb{P}^{3}$ of degree strictly less than $d$.

REMARK. $\mathscr{C}_{L . d}$ is a rather complicated subset of $\mathbb{P}\left(W_{L}^{4}\right)$. It would be interesting to determine the structure of this space.

It follows from (2.1) that branched superminimal immersions of $\Sigma$ into $S^{4}$ of area $4 \pi d$ and arising from the line bundle $L$ are parametrized by the quasi-projective variety

$$
\mathscr{S}_{L, d}:=\mathscr{M}_{L, d} \backslash \mathscr{C}_{L, d}
$$

REMARK. Given $\left[s_{0}, s_{1}, s_{2}, s_{3}\right] \in \mathscr{S}_{L, d}$, we allow one of the pair of pencils (but not both), $\left\langle s_{0}, s_{1}\right\rangle$ or $\left\langle s_{2}, s_{3}\right\rangle$, to have base-points. At such a base point, the corresponding curve $\left[s_{0}, s_{1}, s_{2}, s_{3}\right]$ in $\mathbb{C} \mathbb{P}^{3}$ intersects one of the lines $L_{0}$ or $L_{1}$, hence the corresponding associated meromorphic function has degree less than $d$. Thus, in the genus zero case, $\mathscr{S}_{\mathscr{O}(d)}$ contains elements which lie in the boundary of the moduli space described in [L]. 
Let $\mathscr{B}_{L}:=\left\{(s, t) \in W_{L}^{2} \mid \operatorname{Ram}(s, t)=0\right\}$. Observe that $(s, t) \in \mathscr{B}_{L}$ if and only if $s=\lambda t$ for some constant $\lambda \in \mathbb{C}$, that is, $s \wedge t=0$. Thus we can write

$$
\mathscr{B}_{L}=\left\{(a \sigma, b \sigma) \in W_{L}^{2} \mid \sigma \in W_{L},[a, b] \in \mathbb{C P}^{1}\right\} .
$$

It follows from (2.4) and (2.7) that the fibre above $0 \in H^{0}\left(\Sigma, K L^{2}\right)$ in the fibre product $W_{L}^{2} \times_{H^{0}\left(\Sigma, K L^{2}\right)} W_{L}^{2}$ is just the set

$$
\mathscr{B}_{L} \times \mathscr{B}_{L}=\left\{(a \sigma, b \sigma, c \tau, d \tau) \mid(\sigma, \tau) \in W_{L}^{2}, \quad[a, b],[c, d] \in \mathbb{C P}^{1}\right\} .
$$

Let us now consider the set $\mathbb{P}\left(\mathscr{B}_{L} \times \mathscr{B}_{L}\right)$. Fix $([a, b],[c, d]) \in \mathbb{C P}^{1} \times \mathbb{C P}^{1}$ and let $\ell$ denote the line in $\mathbb{C P}^{3}$ spanned by $[a, b, 0,0] \in L_{0}$ and $[0,0, c, d] \in L_{1}$. Note that $\ell$ is horizontal since the map $\psi$ in (1.2) has contact fibres. Also, observe that $\ell$ projects to a totally geodesic 2 -sphere $S_{\ell}$ which passes through the north and south poles of $S^{4}$. Now fix $(\sigma, \tau) \in W_{L}^{2}$ such that $\langle\sigma, \tau\rangle$ is base-point-free and set $\phi=[(a \sigma, b \sigma, c \tau, d \tau)] \in \mathbb{P}\left(\mathscr{B}_{L} \times \mathscr{B}_{L}\right)$. Then $\phi=[\sigma(a, b, 0,0)+\tau(0,0, c, d)]$ and hence $\phi(\Sigma) \subset \ell$, that is, $\phi: \Sigma \rightarrow \ell$ is a branched covering map. Thus, the space $\mathbb{P}\left(\mathscr{B}_{L} \times \mathscr{B}_{L}\right) \backslash \mathscr{C}_{L, d}$ parametrizes a family of contact curves in $\mathbb{C P}^{3}$ which project to totally geodesic 2-spheres in $S^{4}$ which pass through the north and south poles of $S^{4}$. Fixing the line $\ell$ in $C P^{3}$ amounts to fixing a totally geodesic 2 -sphere $S_{\ell}$ and the composition $\pi \circ \phi$ is just a branched covering map $\Sigma \rightarrow S_{\ell}$.

Note that for $d>2 g-2, \operatorname{dim} W_{L}=\operatorname{dim} H^{0}(\Sigma, L)=d-g+1$ and thus from (2.7) and (2.8) we have $\operatorname{dim} \mathscr{B}_{L}=d-g+2$, and hence

$$
\operatorname{dim}\left(\mathbb{P}\left(\mathscr{B}_{L} \times \mathscr{B}_{L}\right)\right)=2 d-2 g+3 .
$$

REMARK. In the genus zero case, since elements of $\mathbb{P}\left(\mathscr{B}_{L} \times \mathscr{B}_{L}\right)$ are curves in $\mathbb{C P}^{3}$ which intersect $L_{0} \cup L_{1}$, they are elements in the boundary of the moduli space described in [L].

Now consider the case when $\operatorname{Ram}(s, t) \neq 0$, that is, $s \wedge t \neq 0$. (Note that for a function $f=[s, t]$, we have $s \wedge t \neq 0$.) Since $[s, t] \in \mathbb{P}\left(W_{L}^{2} \backslash \mathscr{B}_{L}\right)$ implies that $s \wedge t \neq 0$, we may consider $[s, t]$ as a projectivized ordered 2-frame in $W_{L}$. Then, the projectivized Stiefel manifold of ordered 2-frames in $W_{L}, \mathbb{P} V\left(2, W_{L}\right)$, is just the set $\mathbb{P}\left(W_{L}^{2} \backslash \mathscr{B}_{L}\right)$. Now, consider the action

$$
\begin{gathered}
\operatorname{PGL}(2, \mathbb{C}) \times \mathbb{P} V\left(2, W_{L}\right) \longrightarrow \mathbb{P} V\left(2, W_{L}\right) \text { defined by } \\
\left(\left(\begin{array}{ll}
a & b \\
c & d
\end{array}\right),[s, t]\right)
\end{gathered}
$$

Let $\left[s \wedge t\right.$ ] denote the 2-plane in $W_{L}$ spanned by $s$ and $t$. Observe that the principal $\operatorname{PGL}(2, \mathbb{C})$-bundle over the Grassmannian $G\left(2, W_{L}\right)$ is given by

$$
\mathbb{P} V\left(2, W_{L}\right) \stackrel{p}{\rightarrow} G\left(2, W_{L}\right) \text { where }[s, t] \mapsto[s \wedge t] .
$$


Given a line bundle $\mathscr{L} \rightarrow \Sigma$ and a holomorphic section $\alpha \in H^{0}(\Sigma, \mathscr{L})$, we let $(\alpha)$ denote the divisor of $\alpha$. We have an induced map

$$
(\mathrm{Ram}): \mathbb{P} V\left(2, W_{L}\right)=\mathbb{P}\left(W_{L}^{2} \backslash \mathscr{B}_{L}\right) \longrightarrow \mathbb{P} H^{0}\left(\Sigma, K L^{2}\right)
$$

defined by sending a point $[s, t]$ to the divisor $(s d t-t d s)$. A simple computation shows that the ramification map (2.10) factors through the Grassmannian, that is, the following diagram commutes:

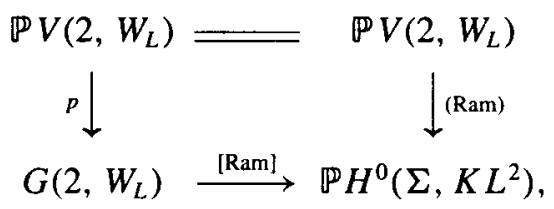

where the map [Ram] is given by $[s \wedge t] \mapsto(s d t-t d s)$. (This is independent of the choice of spanning vectors of the 2-plane.) Consider the Plücker embedding $G\left(2, W_{L}\right) \hookrightarrow \mathbb{P}\left(\bigwedge^{2} W_{L}\right)$. Recall equation (2.3):

$$
\Lambda: \bigwedge^{2} W_{L} \longrightarrow H^{0}\left(\Sigma, K L^{2}\right) .
$$

Projectivizing (2.12), we obtain a rational map:

$$
\bar{\Lambda}: \mathbb{P} \bigwedge^{2} W_{L} \rightarrow \mathbb{P} H^{0}\left(\Sigma, K L^{2}\right),
$$

which when restricted to the image of the Grassmannian under the Plücker embedding (the projectivized simple bivectors) is just the map [Ram] in (2.11). Observe that since $\Lambda(v \wedge w)=0$ implies that $v \wedge w=0$, the base locus of the map $\bar{\Lambda}$ does not intersect the Plücker image of the Grassmannian. We next show that $\bar{\Lambda}$ restricted to the Plücker image of the Grassmannian is a finite map onto its image. We let $G\left(2, W_{L}\right)$ denote the Plücker image of the Grassmannian in $\mathbb{P} \wedge^{2} W_{L}$.

LEMMA 2.1. Let $L$ be a line bundle of degree $d$ over a compact Riemann surface $\Sigma$ of genus $g$. Let $W_{L}=H^{0}(\Sigma, L)$, and suppose that $\operatorname{dim} W_{L} \geq 2$. Then the map $\bar{\Lambda}$ restricted to $G\left(2, W_{L}\right)$ is a finite map onto its image.

Proof. Let $\mu: A \rightarrow B$ be a linear map between two vector spaces and let $Z$ denote its kernel. Then $A=Z \oplus A / Z$ and hence, up to a choice of coordinates, $\mu$ is just the second factor projection in $A$. The corresponding rational map from $\mathbb{P} A$ to $\mathbb{P} B$ is then nothing but the projection map in $\mathbb{P} A$ from the base locus $\mathbb{P} Z$ (up to a choice of coordinates). Thus the rational map $\bar{\Lambda}$ in (2.13) can be considered a projection map from the base locus. Since the Grassmannian does not intersect the base locus, we see that the fibre of the projection map containing a point $x$ in the complement of the base 
locus is just the span of $x$ with the base locus. Thus, by definition of the projection map, the base locus has codimension one in the fibre. Observe that the intersection of this fibre with the Grassmannian cannot have positive dimension since otherwise it will contain a projective line which will intersect the base locus. This implies that $\bar{\Lambda}$ restricted to the Grassmannian is a finite map.

The above lemma is in fact a special case of a more general result:

Proposition 2.2. Let $G$ denote the Grassmannian of $k$-planes in $\mathbb{C}^{m}$ and let $f$ : $G \rightarrow \mathbb{C P}^{n}$ be a nontrivial holomorphic map. Then $f$ is a finite map onto its image.

PrOOF. Consider the ample line bundle $\mathscr{L}$ on $G$ which gives the Plücker embedding of $G$ into $\mathbb{P}\left(\bigwedge^{k} \mathbb{C}^{m}\right)$. Observe that $\mathscr{L}$ is a generator of $\operatorname{Pic}(G)$. Then $f^{*}(\mathscr{O}(1))=\mathscr{L}^{N}$ for some integer $N$. Since $f$ is non-constant, $N \neq 0$. Since $\mathscr{O}(1)$ is ample, we can pull back nontrivial sections to $f^{*}(\mathscr{O}(1))$ and hence $N$ is nonnegative. Therefore $N>0$ and $\mathscr{L}^{N}$ is ample. Suppose $f$ has a positive dimensional fibre, say $F$. Then $\left.\mathscr{L}^{N}\right|_{F}$ is trivial, contradicting ampleness. Thus, $f$ is finite.

PROPOSITION 2.3. Let $L$ be a line bundle of degree $d$ over a compact Riemann surface $\Sigma$ of genus $g$. Let $W_{L}=H^{0}(\Sigma, L)$, and suppose that $\operatorname{dim} W_{L} \geq 3$. Then the map $[\mathrm{Ram}]: G\left(2, W_{L}\right) \rightarrow[\operatorname{Ram}]\left(G\left(2, W_{L}\right)\right) \subset \mathbb{P} H^{0}\left(\Sigma, K L^{2}\right)$ is proper. Moreover, the number of points in a general fibre is $N$ for some positive integer $N$.

PROOF. We first show that [Ram] is nonconstant. Let $s_{0}, s_{1}$ and $s_{2}$ be three linearly independent sections in $W_{L}$. Suppose that $s_{0} d s_{1}-s_{1} d s_{0}=s_{0} d s_{2}-s_{2} d s_{0}$. This implies that $s_{0} d s_{3}-s_{3} d s_{0}=0$ where $s_{3}=s_{1}-s_{2}$, and hence $\left[s_{0} \wedge s_{3}\right] \notin G\left(2, W_{L}\right)$, that is, $s_{0} \wedge s_{3}=0$. In other words, $s_{0}, s_{1}$ and $s_{2}$ are not linearly independent, a contradiction.

By Proposition $2.2,[\operatorname{Ram}]^{*} \mathscr{O}(1)=\mathscr{L}^{N}$ for some positive integer $N$. By $[\mathrm{F}$, Proposition 10.2] the general fibre has $N$ points (counting multiplicity).

REMARK 2.4. By upper semi-continuity, special fibres may contain more than $N$ points. However, if the image $[\operatorname{Ram}]\left(G\left(2, W_{L}\right)\right)$ is smooth, then [Ram] is flat and hence every fibre has $N$ points. Observe also that $N$ depends on $L$.

REMARK. If $\Sigma=S^{2}$, then $\operatorname{dim} W_{L}=d+1, \operatorname{dim} \mathbb{P} H^{0}\left(K L^{2}\right)=2 d-2=$ $\operatorname{dim} G\left(2, W_{L}\right)$. Thus, [Ram] : $G\left(2, W_{L}\right) \rightarrow \mathbb{P} H^{0}\left(K L^{2}\right)$ is a holomorphic map between two complex manifolds of the same dimension and is hence a branched covering map. It was shown in [L] that the degree of this map is precisely the degree of $G\left(2, W_{L}\right)$ in $\mathbb{P} \wedge^{2} W_{L}$ under the Plücker embedding. Thus every fibre of [Ram] has $(2 d-2) ! /[d !(d-1) !]$ points, counting multiplicity. 
Recall that if $\operatorname{deg} L=d>2 g-2$, then $\operatorname{dim} W_{L}=d-g+1$ and so the requirement that $\operatorname{dim} W_{L} \geq 3$ implies that $d \geq g+2$. Henceforth, we shall suppose that $d \geq \max \{2 g-1, g+2\}$. Consider the diagram

$$
\begin{aligned}
& G\left(2, W_{L}\right) \times G\left(2, W_{L}\right) \\
&\left.\right|_{[\operatorname{Ram}] \times[\mathrm{Ram}]} \\
& \mathbb{P} H^{0}\left(\Sigma, K L^{2}\right) \stackrel{\Delta}{\longrightarrow} \mathbb{P} H^{0}\left(\Sigma, K L^{2}\right) \times \mathbb{P} H^{0}\left(\Sigma, K L^{2}\right),
\end{aligned}
$$

where $\Delta$ is the diagonal inclusion. By the finiteness result of Proposition 2.3, we see that the dimension of the fibre product is given by

$$
\operatorname{dim}\left(G\left(2, W_{L}\right) \times_{\mathbb{P} H^{0}\left(\Sigma, K L^{2}\right)} G\left(2, W_{L}\right)\right)=\operatorname{dim}\left(G\left(2, W_{L}\right)\right)=2(d-g-1) .
$$

Observe that the fibre product $\mathbb{P} V\left(2, W_{L}\right) \times_{\mathbb{P} H^{0}\left(\Sigma, K L^{2}\right)} \mathbb{P} V\left(2, W_{L}\right)$ is just the total space of the $(\operatorname{PGL}(2, \mathbb{C}) \times \operatorname{PGL}(2, \mathbb{C}))$-bundle over the fibre product $G\left(2, W_{L}\right) \times_{\mathbb{P} H^{0}\left(\Sigma, K L^{2}\right)}$ $G\left(2, W_{L}\right)$. Let

$$
([s, t],[u, v]) \in \mathbb{P} V\left(2, W_{L}\right) \times_{\mathbb{P} H^{0}\left(\Sigma, K L^{2}\right)} \mathbb{P} V\left(2, W_{L}\right) .
$$

Since

$$
[\operatorname{Ram}]([s \wedge t])=[\operatorname{Ram}]([u \wedge v]) \in \mathbb{P} H^{0}\left(\Sigma, K L^{2}\right),
$$

there exists a unique $\lambda^{2} \in \mathbb{C} \backslash\{0\}$ such that

$$
\operatorname{Ram}(s, t)=-\lambda^{2} \operatorname{Ram}(u, v)=-\operatorname{Ram}( \pm \lambda u, \pm \lambda v),
$$

thereby giving us a pair $[s, t, \pm \lambda u, \pm \lambda v] \in \mathscr{M}_{L, d}$. This gives us a 2:1 correspondence

$$
\begin{aligned}
\mathscr{M}_{L, d} \backslash \mathbb{P}\left(\mathscr{B}_{L} \times \mathscr{B}_{L}\right) & \longrightarrow \mathbb{P} V\left(2, W_{L}\right) \times_{\mathbb{P} H^{0}\left(\Sigma, K L^{2}\right)} \mathbb{P} V\left(2, W_{L}\right) \text { given by } \\
{[s, t, \pm \lambda u, \pm \lambda v] } & \longmapsto([s, t],[u, v]) .
\end{aligned}
$$

This $2: 1$ correspondence is directly related to the $2: 1$ branched covering map $\tilde{\psi}$ in (1.3), and the contact involution discussed in [L].

REMARK 2.5. Let $G=(\operatorname{SL}(2, \mathbb{C}) \times \operatorname{SL}(2, \mathbb{C})) / \pm 1$, where $1=(I, I)$.

1. $G$ acts on $\mathscr{M}_{L, d}$ and leaves the set $\mathbb{P}\left(\mathscr{B}_{L} \times \mathscr{B}_{L}\right)$ invariant.

2. $G$ acts freely on $\mathscr{M}_{L, d} \backslash \mathbb{P}\left(\mathscr{B}_{L} \times \mathscr{B}_{L}\right)$. In fact

$$
\mathscr{M}_{L, d} \backslash \mathbb{P}\left(\mathscr{B}_{L} \times \mathscr{B}_{L}\right) \longrightarrow G\left(2, W_{L}\right) \times_{\mathbf{p}_{H^{0}}\left(\Sigma, K L^{2}\right)} G\left(2, W_{L}\right)
$$

given by

$$
\left[s_{0}, s_{1}, s_{2}, s_{3}\right] \longmapsto\left(\left[s_{0} \wedge s_{1}\right],\left[s_{2} \wedge s_{3}\right]\right)
$$

is a principal $G$-bundle. 
3. $G \longrightarrow \operatorname{PGL}(2, \mathbb{C}) \times \operatorname{PGL}(2, \mathbb{C})$ is a $2: 1$ covering map and thus so is

$$
\mathscr{M}_{L, d} \backslash \mathbb{P}\left(\mathscr{B}_{L} \times \mathscr{B}_{L}\right) \longrightarrow \mathbb{P} V\left(2, W_{L}\right) \times_{\mathbb{P} H^{0}\left(\Sigma, K L^{2}\right)} \mathbb{P} V\left(2, W_{L}\right) .
$$

Thus,

$$
\operatorname{dim} \mathscr{S}_{L, d}=\operatorname{dim} \mathscr{M}_{L, d}=2(d-g-1)+6=2(d-g+2)
$$

since $\operatorname{dim} G=6$. Observe from (2.9) that $\mathbb{P}\left(\mathscr{B}_{L} \times \mathscr{B}_{L}\right)$ is of codimension 1 in $\mathscr{M}_{L, d}$.

\section{The moduli space via bundles over the Jacobian variety}

Let $J_{d}$ denote the Jacobian variety of equivalence classes of degree $d$ line bundles over $\Sigma$ (also denoted $\operatorname{Pic}^{d}(\Sigma)$ ). For each $j \in J_{d}$, let $L_{j}$ denote the line bundle corresponding to $j$, and let $W_{j}=H^{0}\left(\Sigma, L_{j}\right)$.

REMARK 3.1. When $d>2 g-2, \operatorname{dim} W_{j}=d-g+1$ for all $j \in J_{d}$. Henceforth, we shall assume that $d \geq \max \{2 g-1, g+2\}$.

Recall that there exists a line bundle, $\mathscr{P} \longrightarrow \Sigma \times J_{d}$, called the Poincare line bundle, with the property that $\left.\mathscr{P}\right|_{\Sigma \times\{\}} \cong L_{j}$ (see [ACGH] for example). Let $\pi: \Sigma \times J_{d} \rightarrow J_{d}$ denote the second factor projection. Consider the direct image sheaf $\pi_{*} \mathscr{P} \rightarrow J_{d}$. Observe that

$$
\left.\pi_{*} \mathscr{P}\right|_{j} \cong H^{0}\left(\Sigma \times\{j\},\left.\mathscr{P}\right|_{\Sigma \times(j\}}\right) \cong H^{0}\left(\Sigma, L_{j}\right)=W_{j} .
$$

As a set, $\pi_{*} \mathscr{P}=\bigcup_{j \in J_{d}} W_{j}$. By Remark 3.1, since $d>2 g-2$, each fibre is of dimension $d-g+1$. In fact, $\pi_{*} \mathscr{P}$ has the structure of a complex vector bundle of rank $d-g+1$ and it is called a Picard bundle. For simplicity, we let $\mathscr{W}=\pi_{*} \mathscr{P}$. Given a vector bundle $\xi$, we shall let $\mathscr{P} \xi$ denote the projective bundle of lines through the origin of the fibres of $\xi$. We consider the following associated bundles and sub-bundles over $J_{d}$ :

1. $\mathscr{W}^{k}$, the Whitney sum of $k$ copies of $\mathscr{W}$;

2. the projective bundle $\mathscr{P}^{2} \mathscr{W}^{k}$;

3. the rank $d-g+2$ sub-bundle, $\mathscr{B}$, of $\mathscr{W}^{2}$, with fibre isomorphic to $\mathscr{B}_{L_{j}}$;

4. the projective bundle $\mathscr{P}(\mathscr{B} \oplus \mathscr{B})$, of rank $2 d-2 g+3$ with fibre isomorphic to $\mathbb{P}\left(\mathscr{B}_{L_{j}} \times \mathscr{B}_{L_{j}}\right)$

5. the bundle $\bigwedge^{2} \mathscr{W}\left(=\bigcup_{j \in J_{d}} \bigwedge^{2} W_{j}\right.$ as a set $)$;

6. the Grassmann bundle of 2-planes, $\mathscr{G}$, with fibre isomorphic to $G\left(2, W_{j}\right)$;

7. the projective Stiefel bundle, $\mathscr{P} \mathscr{V}$ (this is the principal PGL(2, C)-bundle over $\mathscr{G})$. 
Now consider the Jacobian variety $J_{d+g-1}$. For $j \in J_{d+g-1}$, the corresponding line bundle can be expressed as $\mathscr{L}_{j}=K^{1 / 2} \otimes L_{j}$, where $L_{j}$ is a line bundle of degree $d$, and $K$ is the canonical line bundle over $\Sigma$. Let $\mathscr{P}_{1} \longrightarrow \Sigma \times J_{d+g-1}$ denote the Poincare line bundle with the property that $\left.\mathscr{P}_{1}\right|_{\Sigma \times[j]} \cong \mathscr{L}_{j}=K^{1 / 2} \otimes L_{j}$. Let $\pi: \Sigma \times J_{d+g-1} \rightarrow J_{d+g-1}$ denote the second factor projection and consider the direct image $\pi_{*}\left(\mathscr{P}_{1} \otimes \mathscr{P}_{1}\right) \rightarrow J_{d+g-1}$. Observe that

$$
\left.\pi_{*}\left(\mathscr{P}_{1} \otimes \mathscr{P}_{1}\right)\right|_{j} \cong H^{0}\left(\Sigma \times\{j\},\left.\left(\mathscr{P}_{1} \otimes \mathscr{P}_{1}\right)\right|_{\Sigma \times(j)}\right) \cong H^{0}\left(\Sigma, K \otimes L_{j}^{2}\right) .
$$

Note that $\operatorname{dim} H^{0}\left(\Sigma, K \otimes L_{j}^{2}\right)=(2 d+2 g-2)-g+1=2 d+g-1$ for each $j \in J_{d}$. Let $f: J_{d} \rightarrow J_{d+g-1}$ be defined by $L_{j} \mapsto K^{1 / 2} \otimes L_{j}$. Let $\mathscr{K} \rightarrow J_{d}$ denote the complex vector bundle of rank $2 d+g-1$ defined by $f^{*}\left(\pi_{*}\left(\mathscr{P}_{1} \otimes \mathscr{P}_{1}\right)\right)$. We have a bundle map

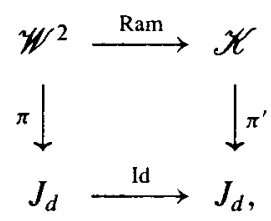

where the top arrow is defined by $(s, t) \mapsto \operatorname{Ram}(s, t)$. Note that the inverse image under Ram of the zero section in $\mathscr{K}$ is just the bundle $\mathscr{B} \rightarrow J_{d}$. Consider the diagonal inclusion of $\mathscr{K}$ into the Whitney sum $\mathscr{K} \oplus \mathscr{K}$ :

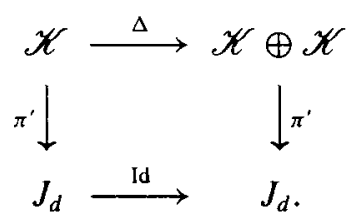

Next, from the bundle maps over $J_{d}$ :

$$
\begin{aligned}
& \mathscr{W}^{2} \oplus \mathscr{W}^{2}=\mathscr{W}^{4} \\
& \lfloor\operatorname{Ram} \oplus\{-\operatorname{Ram}\} \\
& \mathscr{K} \stackrel{\Delta}{\longrightarrow} \quad \mathscr{K} \oplus \mathscr{K},
\end{aligned}
$$

where the maps on the base $J_{d}$ are identity maps, consider the projectivized fibre product

$$
\mathscr{M}_{d, \Sigma}:=\mathscr{P}\left(\mathscr{W}^{2} \times \mathscr{X} \mathscr{W}^{2}\right) \subset \mathscr{P} \mathscr{W}^{4},
$$

that is, the fibrewise projectivization of the fibre product $\mathscr{W}^{2} \times \mathscr{X} \mathscr{W}^{2} \subset \mathscr{W}^{4}$ (over $J_{d}$ ). Notice that the restriction of $\mathscr{M}_{d, \Sigma}$ to the zero section in $\mathscr{K}$ is just the bundle $\mathscr{P}(\mathscr{B} \times \mathscr{B}) \rightarrow J_{d}$. Recall that

$$
\mathscr{C}_{L_{j}, d}:=\left\{\left[s_{0}, s_{1}, s_{2}, s_{3}\right] \in \mathbb{P}\left(W_{j}^{4}\right) \mid\left\langle s_{0}, s_{1}, s_{2}, s_{3}\right\rangle \text { has base-points }\right\} .
$$

Let $\mathscr{C}_{d, \Sigma}:=\bigcup_{j \in J_{d}} \mathscr{C}_{L_{j}, d} \subset \mathscr{P} \mathscr{W}^{4}$. 
REMARK 3.2. Branched superminimal immersions of $\Sigma$ into $S^{4}$ of area $4 \pi d$ are parametrized by the quasi-projective variety

$$
\mathscr{S}_{d, \Sigma}:=\mathscr{M}_{d, \Sigma} \backslash \mathscr{C}_{d, \Sigma}
$$

Observe that the projective Stiefel bundle over $J_{d}$ is just the bundle with fibre $\mathbb{P}\left(W_{j}^{2} \backslash \mathscr{B}_{L_{j}}\right)$. Projectivizing the bundle $\mathscr{K}$ fibrewise, we have an associated $\left(\mathbb{P}^{2 d+g-2}\right)$-bundle, $\mathscr{P} \mathscr{K} \stackrel{\pi^{\prime}}{\rightarrow} J_{d}$. The maps (Ram) and [Ram] extend to bundle maps:

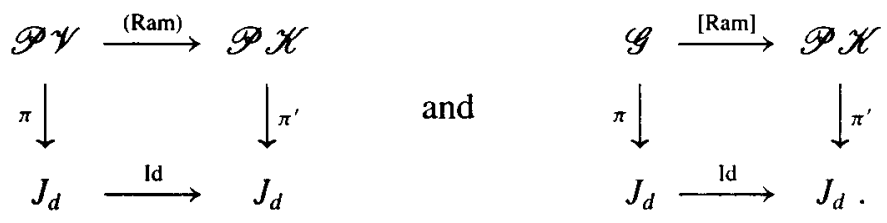

Consider the diagonal inclusion of $\mathscr{P} \mathscr{K}$ into the Whitney sum $\mathscr{P} \mathscr{K} \oplus \mathscr{P} \mathscr{K}$ :

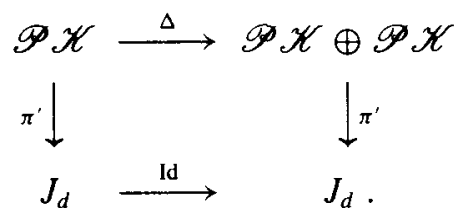

Now, from the diagrams:

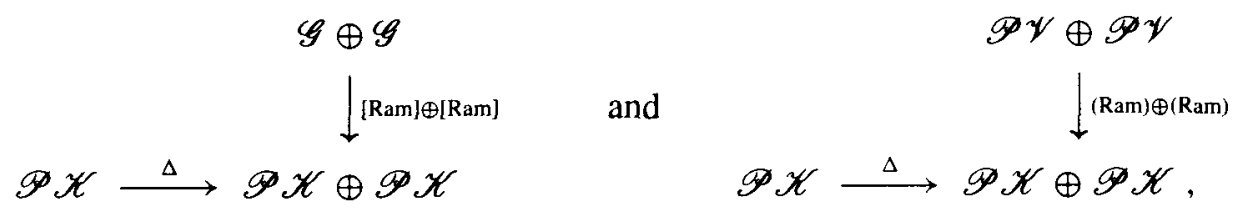

where the maps on the base $J_{d}$ are identity maps, consider the fibre products $\mathscr{G} \times \mathscr{P} \mathscr{X} \mathscr{G}$ and $\mathscr{P} \mathscr{V} \times \mathscr{P} \mathscr{X} \mathscr{P} \mathscr{V}$. Since $\operatorname{dim} J_{d}=g$ we see that

$$
\operatorname{dim} \mathscr{G} \times_{\mathscr{P} \mathscr{X}} \mathscr{G}=\operatorname{dim} \mathscr{G}=(2 d-2 g-2)+g=2 d-g-2
$$

and so $\operatorname{dim} \mathscr{P} \mathscr{V} \times \mathscr{P} \mathscr{X} \mathscr{P} \mathscr{V}=2 d-g-2+6=2 d-g+4$. Thus,

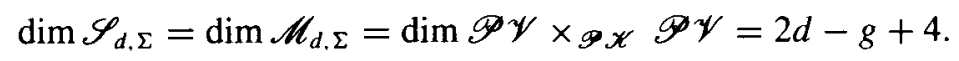

\section{Path-connectedness}

Let us first fix a line bundle $L$ of degree $d$ over $\Sigma$. We claim that $\mathscr{S}_{L, d}$ is pathconnected. 
We first show that any point in $\mathscr{S}_{L, d}$ can be connected by a path to a point in the path-connected space $\mathbb{P}\left(\mathscr{B}_{L} \times \mathscr{B}_{L}\right) \backslash \mathscr{C}_{L . d}$. Let $\left[s_{0}, s_{1}, s_{2}, s_{3}\right] \in \mathscr{S}_{L . d}$. Suppose that $\left\langle s_{0}, s_{2}\right\rangle$ has a base-point, say $p$. Since $\left\langle s_{0}, s_{1}, s_{2}, s_{3}\right\rangle$ is base-point-free, then either $s_{1}(p) \neq 0$ or $s_{3}(p) \neq 0$. Without loss of generality, suppose that $s_{1}(p) \neq 0$. Consider a path $C \subset \mathscr{S}_{L, d}$ defined by

$$
C(t):=\left[s_{0}+t s_{1}, s_{1}, s_{2}, s_{3}\right]
$$

Then $p$ is not a base-point of $\left\langle s_{0}+t s_{1}, s_{2}\right\rangle$ for $t \neq 0$. Thus, we may assume that $\left\langle s_{0}, s_{2}\right\rangle$ is base-point-free. Consider a path $\gamma \subset \mathscr{S}_{L . d}$ defined by

$$
\gamma(t):=\left[s_{0},(1-t) s_{0}+t s_{1}, s_{2},(1-t) s_{2}+t s_{3}\right]
$$

with $\gamma(1)=\left[s_{0}, s_{1}, s_{2}, s_{3}\right]$ and $\gamma(0)=\left[s_{0}, s_{0}, s_{2}, s_{2}\right] \in \mathbb{P}\left(\mathscr{B}_{L} \times \mathscr{B}_{L}\right) \backslash \mathscr{C}_{L, d}$. We now show that $\mathbb{P}\left(\mathscr{B}_{L} \times \mathscr{B}_{L}\right) \backslash \mathscr{C}_{L, d}$ is path-connected. Observe that any point $\left[a \sigma_{0}, b \sigma_{0}, c \sigma_{2}, d \sigma_{2}\right] \in \mathbb{P}\left(\mathscr{B}_{L} \times \mathscr{B}_{L}\right) \backslash \mathscr{C}_{L, d}$ is path-connected to the point $\left[\sigma_{0}, \sigma_{0}, \sigma_{2}, \sigma_{2}\right]$ via a path in $\mathbb{P}\left(\mathscr{B}_{L} \times \mathscr{B}_{L}\right) \backslash \mathscr{C}_{L, d}$. Let $\left[s_{0}, s_{0}, s_{2}, s_{2}\right]$ be another point in $\mathbb{P}\left(\mathscr{B}_{L} \times \mathscr{B}_{L}\right) \backslash \mathscr{C}_{L, d}$. For a given $p \in \Sigma$, let $W_{p}=\left\{s \in W_{L} \mid s(p)=0\right\}$. Observe that the map $W_{L} \rightarrow \mathbb{C}$ given by $s \mapsto s(p)$ is a linear map.

REMARK. If $d \geq 2 g$, then the complete linear system $\mathbb{P} W_{L}$ has no base-points and thus $W_{p}$ is a complex hyperplane in $W_{L}$. Since it is a real codimension two subspace of $W_{L}$, it cannot disconnect $W_{L}$. Henceforth, we shall assume that $d \geq 2 g$.

Let $A=\left\{p \in \Sigma \mid s_{2}(p)=0\right\}$ and consider the finite union of complex hyperplanes in $W_{L}: \mathscr{A}=\bigcup_{p \in A} W_{p}$. Since $\mathscr{A}$ is of real codimension two, there is a path in $W_{L} \backslash \mathscr{A}$ which connects $s_{0}$ and $\sigma_{0}$. Thus, there is a path in $\mathbb{P}\left(\mathscr{B}_{L} \times \mathscr{B}_{L}\right) \backslash \mathscr{C}_{L, d}$ which connects $\left[s_{0}, s_{0}, s_{2}, s_{2}\right]$ to $\left[\sigma_{0}, \sigma_{0}, s_{2}, s_{2}\right]$. Now let $A^{\prime}=\left\{p \in \Sigma \mid \sigma_{0}(p)=0\right\}$ and let $\mathscr{A}^{\prime}=\bigcup_{p \in A^{\prime}} W_{p}$. By a similar argument, we can join $s_{2}$ to $\sigma_{2}$ via a path in $W_{L} \backslash \mathscr{A}^{\prime}$, and thus there is a path in $\mathbb{P}\left(\mathscr{B}_{L} \times \mathscr{B}_{L}\right) \backslash \mathscr{C}_{L, d}$ which connects the points $\left[\sigma_{0}, \sigma_{0}, s_{2}, s_{2}\right]$ and $\left[\sigma_{0}, \sigma_{0}, \sigma_{2}, \sigma_{2}\right]$. This proves that the space $\mathbb{P}\left(\mathscr{B}_{L} \times \mathscr{B}_{L}\right) \backslash \mathscr{C}_{L, d}$ is path-connected.

THEOREM 4.1. For $d \geq \max \{2 g, g+2\}, \mathscr{S}_{d, \Sigma}$ is a path-connected quasi-projective variety of dimension $2 d-g+4$.

PROOF. We have shown that $\mathscr{S}_{L, d}$ is path-connected for each line bundle $L$ of degree $d \geq \max \{2 g, g+2\}$. Path-connectedness of $\mathscr{S}_{d, \Sigma}$ follows from the fact that $J_{d}$ is a connected Abelian variety. Quasi-projectiveness follows since projective bundles over projective varieties are projective. The dimension comes from (3.3). 


\section{Compactifying}

Recall that $\mathscr{S}_{d, \Sigma}:=\mathscr{M}_{d, \Sigma} \backslash \mathscr{C}_{d, \Sigma}$, where $\mathscr{M}_{d, \Sigma}:=\mathscr{P}\left(\mathscr{W}^{2} \times \mathscr{X} \mathscr{W}^{2}\right) \subset \mathscr{P}^{4} \mathscr{W}^{4}$. Since $\mathscr{M}_{d, \Sigma}$ is compact, it is an obvious compactification of $\mathscr{S}_{d, \Sigma}$. Unfortunately, elements of $\mathscr{C}_{d, \Sigma}$ correspond to parametrized curves of degree strictly less than $d$. Thus, from the geometric viewpoint, $\mathscr{M}_{d . \Sigma}$ is not a satisfactory compactification of the moduli space, $\mathscr{S}_{d, \Sigma}$, of branched superminimal immersions of $\Sigma$ into $S^{4}$ with area $4 \pi d$. In this section, we shall consider the genus zero case, that is, when $\Sigma=\mathbb{C P}^{1}$. (Hence, $J_{d}$ has just one element- $\mathscr{O}(d)$.) We shall let $\mathscr{S}_{d}=\mathscr{S}_{d . \text { CPI }}$.

Since we are considering spaces of maps, it is appropriate to study the corresponding spaces of graphs. Recall that the graph of a map $f: M \rightarrow N$ is the subset $\Gamma_{f}=$ $\{(x, f(x)) \mid x \in M\} \subset M \times N$. In our case, $M=\mathbb{C P}^{1}$ and $N=\mathbb{C P}^{3}$. Let $\pi_{1}: \mathbb{C P}^{1} \times \mathbb{C P}^{3} \rightarrow \mathbb{C P}^{1}$ and $\pi_{2}: \mathbb{C P}^{1} \times \mathbb{C P}^{3} \rightarrow \mathbb{C P}^{3}$ denote the first and second factor projection maps respectively. Let $C$ be a curve in $\mathbb{C P}^{1} \times \mathbb{C P}^{3}$. Since $H_{2}\left(\mathbb{C} \mathbb{P}^{n}, \mathbb{Z}\right)=\mathbb{Z}$, we have a well-defined notion of homology degree for $\pi_{i}(C), i=1,2$. We say that $C$ has bidegree $(m, n)$ if $\operatorname{deg}\left(\pi_{1}(C)\right)=m$ and $\operatorname{deg}\left(\pi_{2}(C)\right)=n$. Thus, each element $s \in \mathscr{S}_{d}$ corresponds to a smooth curve $\Gamma_{s} \subset \mathbb{C P}^{1} \times \mathbb{C P}^{3}$ of bidegree $(1, d)$. Let $\mathscr{H}_{1 . d}$ denote the Hilbert scheme of rational curves of bidegree $(1, d)$ in $\mathbb{C P}^{1} \times \mathbb{C P}^{3}$ and let $\mathscr{G}_{1, d} \subset \mathscr{H}_{1, d}$ denote the component of the Hilbert scheme containing the graphs of degree $d$ maps of $\mathbb{C P}^{1}$ to $\mathbb{C P}^{3}$. We have a map $\Gamma: \mathscr{S}_{d} \rightarrow \mathscr{G}_{1 . d}$ defined by $s \mapsto \Gamma_{s}$. The desired compactification is then given by the closure of $\Gamma\left(\mathscr{S}_{d}\right)$ in $\mathscr{G}_{1, d}$. We shall call the elements of the boundary 'limit graphs'. The question is: what do the limit graphs look like? This is a rather difficult question. Nevertheless, we expect a limit graph to be a curve, not necessarily irreducible, of bidegree $(1, d)$.

Let $z_{0}, z_{1}$ and $x_{0}, x_{1}, x_{2}, x_{3}$ denote the homogeneous variables for $\mathbb{C P} 1$ and $\mathbb{C P}^{3}$ respectively. Let $S_{z}^{n}$ and $S_{x}^{n}$ denote the space of homogeneous polynomials of degree $n$ in the variables $z_{0}, z_{1}$, and the variables $x_{0}, x_{1}, x_{2}, x_{3}$ respectively. Since $H^{0}\left(\mathbb{C} \mathbb{P}^{1}, \mathscr{O}(d)\right)=S_{z}^{d}$, any parametrized rational curve of degree $d$ in $\mathbb{C P}^{3}$ is of the form $s=\left[s_{0}, s_{1}, s_{2}, s_{3}\right]$ where $s_{i} \in S_{z}^{d}$ for $i=0, \ldots, 3$, and $\operatorname{gcd}\left(s_{0}, s_{1}, s_{2}, s_{3}\right)=1$. (Elements in $\mathscr{S}_{d}$ have the additional property that $\operatorname{Ram}\left(s_{0}, s_{1}\right)=-\operatorname{Ram}\left(s_{2}, s_{3}\right)$.) The graph $\Gamma_{s} \subset \mathbb{C P}^{1} \times \mathbb{C P}^{3}$ is defined by 6 bihomogeneous equations:

$$
F_{i, j}:=s_{i} x_{j}-s_{j} x_{i}=0, \quad \text { where } i<j, \quad i=0,1,2, \quad j=1,2,3 .
$$

Observe that the $F_{i, j}$ 's are elements of $S_{z}^{d} S_{x}^{1}$, the tensor product of $S_{z}^{d}$ and $S_{x}^{1}$. From (5.1) we see that in general $\Gamma_{s}$ is not a complete intersection. This makes the study of the boundary elements much more difficult. An obvious way of compactifying the space of graphs is by dropping the gcd condition. (This corresponds to the inclusion of $\mathscr{C}_{d}$ in our space.) One approach to understanding limit graphs is to consider 1-parameter families. More precisely, consider a curve $\varphi_{t}$ in $\mathscr{M}_{d}$ such that $\varphi_{0}=c \in \mathscr{C}_{d}$. Suppose that $\varphi_{t} \backslash\{c\} \subset \mathscr{S}_{d}$. Then $\varphi_{t} \backslash\{c\}$ parametrizes a flat family of curves in $\mathbb{C P}^{1} \times \mathbb{C P}^{3}$. 
Since flatness extends uniquely across a puncture, we obtain a unique limit graph associated to the curve $\varphi_{t}$. Let us first consider the following simple example.

EXAMPLE 5.1. Let $\varphi_{t}=\left[z_{0}, z_{0}, z_{0}+t z_{1}, z_{0}+t z_{1}\right]$. Note that $\varphi_{t} \in \mathscr{S}_{1}$ for $t \neq 0$ and $\varphi_{0} \in \mathscr{C}_{1}$. The equations for $\Gamma_{\varphi}$ are obtained by setting each of the following six polynomials equal to zero:

$$
\begin{array}{ll}
F_{0,1}^{t}:=z_{0}\left(x_{1}-x_{0}\right), & F_{0,2}^{t}:=z_{0}\left(x_{2}-x_{0}\right)-t z_{1} x_{0}, \\
F_{0,3}^{t}:=z_{0}\left(x_{3}-x_{0}\right)-t z_{1} x_{0}, & F_{1,2}^{t}:=z_{0}\left(x_{2}-x_{1}\right)-t z_{1} x_{1}, \\
F_{1,3}^{t}:=z_{0}\left(x_{3}-x_{1}\right)-t z_{1} x_{1}, & F_{2,3}^{t}:=\left(z_{0}+t z_{1}\right)\left(x_{3}-x_{2}\right) .
\end{array}
$$

Set $S=\mathbb{C}\left[x_{0}, x_{1}, x_{2}, x_{3}, z_{0}, z_{1}\right]$. For each constant $t$, let $I_{t}=\left(F_{i, j}^{t}\right) \subset S$ denote the ideal generated by the 6 bihomogeneous polynomials defined in (5.2). Then the graph described by the system of equations obtained by setting the polynomials in (5.2) equal to zero is just the variety $V\left(I_{t}\right)$ for $t \neq 0$. It is tempting to say that the limit as $t$ approaches 0 of $\Gamma_{\varphi_{t}}=V\left(I_{t}\right)$ is the variety $V\left(I_{0}\right)$. However, if we simply set $t=0$ in the system of equations obtained from (5.2), we obtain

$$
V\left(I_{0}\right)=\mathbb{C} \mathbb{P}^{1} \times\{[1,1,1,1]\} \cup\{[0,1]\} \times \mathbb{C P}^{3} \subset \mathbb{C P}^{1} \times \mathbb{C P}^{3} .
$$

Observe that the $\{[0,1]\} \times \mathbb{C} \mathbb{P}^{3}$ term above is of dimension three. Since this notion of a limit of curves obtained via a limit of equations does not preserve dimension, it is not satisfactory from the geometric viewpoint. We have to look for an alternative approach for taking limits. We would like to complete the picture in (5.3) by 'filling in' a curve in the second factor of the $\{[0,1]\} \times \mathbb{C P}^{3}$ term. To do this, we need to enlarge the ideal $I_{t}$ via an algebraic process called saturation. We proceed as follows. By setting each of the polynomials in (5.2) equal to zero we obtain the following system of equations:

$$
\begin{array}{rlrl}
F_{0,1}^{t}\left(x_{i}-x_{0}\right)-F_{0, i}^{t}\left(x_{1}-x_{0}\right) & =t z_{1} x_{0}\left(x_{1}-x_{0}\right), & & i=2,3 ; \\
F_{0,1}^{t}\left(x_{i}-x_{1}\right)-F_{1, i}^{t}\left(x_{1}-x_{0}\right) & =t z_{1} x_{1}\left(x_{1}-x_{0}\right), & i=2,3 ; \\
F_{1, i}^{t}\left(x_{i}-x_{0}\right)-F_{0, i}^{t}\left(x_{i}-x_{1}\right) & =t z_{1} x_{i}\left(x_{1}-x_{0}\right), & i=2,3 ; \\
F_{1,2}^{t}\left(x_{3}-x_{0}\right)-F_{0,3}^{t}\left(x_{2}-x_{1}\right) & =t z_{1}\left[x_{0} x_{2}-x_{1} x_{3}\right] ; & \\
F_{1,3}^{t}\left(x_{2}-x_{0}\right)-F_{0,2}^{t}\left(x_{3}-x_{1}\right) & =t z_{1}\left[x_{0} x_{3}-x_{1} x_{2}\right] ; & \\
F_{2,3}^{t}\left(x_{1}-x_{0}\right)-F_{0,1}^{t}\left(x_{3}-x_{2}\right) & =t z_{1}\left(x_{3}-x_{2}\right)\left(x_{1}-x_{0}\right) ; \\
F_{2,3}^{t}\left(x_{i}-x_{1}\right)-F_{1, i}^{t}\left(x_{3}-x_{2}\right) & =t z_{1} x_{i}\left(x_{3}-x_{2}\right), \quad i=2,3 .
\end{array}
$$

Consider the polynomials on the right side of each equality of (5.4) with $t=1$. Let these polynomials: $z_{1} x_{0}\left(x_{1}-x_{0}\right), z_{1} x_{1}\left(x_{1}-x_{0}\right), z_{1} x_{2}\left(x_{1}-x_{0}\right), z_{1} x_{3}\left(x_{1}-x_{0}\right)$, $z_{1}\left(x_{0} x_{2}-x_{1} x_{3}\right), z_{1}\left(x_{0} x_{3}-x_{1} x_{2}\right), z_{1} x_{2}\left(x_{3}-x_{2}\right), z_{1} x_{3}\left(x_{3}-x_{2}\right)$ and $z_{1}\left(x_{3}-x_{2}\right)\left(x_{1}-x_{0}\right)$, 
together with the six generators of $I_{t}$ be the generators of an enlarged ideal, call it $J_{t}$. It follows from the system of equations (5.4) that $J_{t}=I_{t}$ for $t \neq 0$, and thus we have equality of the corresponding varieties $V\left(J_{t}\right)=V\left(I_{t}\right)$ when $t \neq 0$. Note that two bihomogeneous ideals define the same subscheme of $\mathbb{C P ^ { 1 }} \times \mathbb{C P}^{3}$ if and only if they induce the same dehomogenizations. Let $l_{t}^{\prime}=\left(F_{i, j}^{t}, z_{1}\left(x_{1}-x_{0}\right), z_{1}\left(x_{3}-x_{2}\right)\right)$. Observe that $I_{t}^{\prime}$ and $J_{t}$ have the same dehomogenizations. From the $z_{1} \neq 0$ chart, we see that the last two generators of $I_{0}^{\prime}$ imply that $x_{0}=x_{1}$ and $x_{2}=x_{3}$, thus giving us a line $L_{1}$ in $\mathbb{C P}^{3}$ spanned by $[1,1,0,0]$ and $[0,0,1,1]$. We thus obtain the limit graph

$$
V\left(I_{0}^{\prime}\right)=\mathbb{C P}^{1} \times\{[1,1,1,1]\} \cup\{[0,1]\} \times L_{1} \subset \mathbb{C P}^{1} \times \mathbb{C P}^{3} .
$$

This is indeed a curve, albeit one with two irreducible components. The first component is of bidegree $(1,0)$, corresponding to a graph of a constant map $z \mapsto[1,1,1,1]$. The second component is a curve of bidegree $(0,1)$, and is therefore not a graph of any map to $\mathbb{C P}^{3}$; rather it is a 'bubble' when we interpret the limit as $t$ approaches 0 of the 1-parameter family of maps $\left[s_{0},(1-t) s_{0}+t s_{1}, s_{2},(1-t) s_{2}+t s_{3}\right]$ as the second factor projection map of the limit graph:

$$
\mathbb{C P}^{1} \times\{[1,1,1,1]\} \cup\{[0,1]\} \times L_{1} \longmapsto \mathbb{C P}^{3} .
$$

Observe that for each $t$ the image of $\varphi_{t}: \mathbb{C P}^{1} \rightarrow \mathbb{C P}^{3}$ lies in the line $L_{1}$ and thus it is not at all surprising that the image of the limit map is $L_{1}$.

Now let us consider what happens when we have a different curve emanating from the same point $\varphi_{0} \in \mathscr{C}_{1}$.

EXAMPLE 5.2. Let $\psi_{t}=\left[z_{0}+t z_{1}, z_{0}, z_{0}, z_{0}+t z_{1}\right]$. Then $\psi_{0}=\varphi_{0}$. Let $L_{2}$ denote the line in $\mathbb{C P}^{3}$ spanned by $[1,0,0,1]$ and $[0,1,1,0]$. Observe that for each $t$ the image of $\psi_{t}: \mathbb{C P}^{1} \rightarrow \mathbb{C P}^{3}$ lies in the line $L_{2}$. The six bihomogeneous polynomials corresponding to $\Gamma_{\psi_{1}}$ are:

$$
\begin{aligned}
& G_{0,1}^{t}:=z_{0}\left(x_{1}-x_{0}\right)+t z_{1} x_{1}, G_{0,2}^{t}:=z_{0}\left(x_{2}-x_{0}\right)+t z_{1} x_{1}, \\
& G_{0,3}^{t}:=\left(z_{0}+t z_{1}\right)\left(x_{3}-x_{0}\right), G_{1,2}^{t}:=z_{0}\left(x_{2}-x_{1}\right), \\
& G_{1,3}^{t}:=z_{0}\left(x_{3}-x_{1}\right)-t z_{1} x_{1}, G_{2,3}^{t}:=z_{0}\left(x_{3}-x_{2}\right)-t z_{1}\left(x_{2}-x_{1}\right) .
\end{aligned}
$$

Proceeding as in Example 5.1, we obtain $I_{t}^{\prime}=\left(G_{i, j}^{t}, z_{1}\left(x_{2}-x_{1}\right), z_{1}\left(x_{3}-x_{0}\right)\right)$ and thus the limit graph is

$$
V\left(I_{0}^{\prime}\right)=\mathbb{C P}^{1} \times\{[1,1,1,1]\} \cup\{[0,1]\} \times L_{2} \subset \mathbb{C P}^{1} \times \mathbb{C P}^{3} .
$$

Observe that this limit graph is distinct from that obtained in Example 5.1 thus illustrating the fact that limits of graphs depend on the choice of a one-parameter family. 
Examples 5.1 and 5.2 generalize as follows. Let $s=\left[s_{0}, s_{1}, s_{2}, s_{3}\right] \in \mathscr{M}_{d}$ such that $\operatorname{gcd}\left(s_{0}, s_{1}, s_{2}, s_{3}\right)=D \in S_{z}^{m}$, for some $m>0$. Then $s_{i}=D \sigma_{i}$, for some $\sigma_{i} \in S_{z}^{d-m}$, $i=0,1,2,3$. Setting

$$
F_{i, j}^{\prime}=\sigma_{i} x_{j}-\sigma_{j} x_{i}, \quad \text { where } i, j \in\{0,1,2\} \text { and } i<j,
$$

we have six bihomogeneous polynomials:

$$
F_{i, j}=D\left(\sigma_{i} x_{j}-\sigma_{j} x_{i}\right)=D F_{i, j}^{\prime}, \text { where } i, j \in\{0,1,2\} \text { and } i<j .
$$

The variety of the ideal generated by the polynomials in (5.6) is a graph of bidegree $(1, d-m)$ in $\mathbb{C P}^{1} \times \mathbb{C P}^{3}$ corresponding to the map given by $\sigma=\left[\sigma_{0}, \sigma_{1}, \sigma_{2}, \sigma_{3}\right]$. Suppose that $p_{1}, \ldots, p_{m}$ are the roots of $D$. Then the variety of the ideal generated by the polynomials in (5.7) is

$$
\Gamma_{\sigma} \cup \bigcup_{i=1}^{m}\left(\left\{p_{i}\right\} \times \mathbb{C P}^{3}\right) \subset \mathbb{C P}^{1} \times \mathbb{C P}^{3} .
$$

In order to complete this picture, we consider a curve $\varphi_{t}$ in $\mathscr{M}_{d}$ such that $\varphi_{0}=s \in \mathscr{C}_{d}$ and $\varphi \backslash\{s\} \subset \mathscr{S}_{d}$. From the ideal $I_{t}=\left(F_{0, i}^{t}, F_{1, j}^{t}, F_{2, k}^{t}, F_{3, l}^{t}\right)$, we perform some algebra to obtain its saturation $I_{t}^{\prime}$. The limit graph, $V\left(I_{0}^{\prime}\right)$, has at least 2 irreducible components. The first component is $\Gamma_{\sigma}$ and the other components have total bidegree $(0, m)$. This process, however, is rather tedious-even in the simple case of a curve of bidegree $(1,1)$ as seen in Examples 5.1 and 5.2.

REMARK. In [LV1], a simpler compactification problem was studied: the variety parametrizing graphs of holomorphic maps from $\mathbb{C P}^{1}$ to $\mathbb{C P}^{1} \times \mathbb{C} \mathbb{P}^{1}$. (The graph of such a map is a complete intersection.) Here a global approach was taken: obtain the compactification via an explicit sequence of blow-ups along smooth centres. The bidegree $(1,1)$ case is described in [LV1], and the bidegree $(2,2)$ case in [LV2]. It is hoped that an understanding of this simpler compactification problem will be of use in the compactification problem for the moduli space of superminimal surfaces in $S^{4}$.

\section{Acknowledgements}

The author thanks M. S. Narasimhan, Israel Vainsencher and Weng Lin for numerous useful conversations. Part of this work was initiated at Universidade Federal do Ceará, Fortaleza, Brazil and the author would like to thank the university for its hospitality, and also to $\mathrm{CNPq}-\mathrm{Brasil}$ for support. A major part of this work was conducted at the International Centre for Theoretical Physics in Trieste, Italy, and the author expresses his gratitude for its hospitality and support. 


\section{References}

[ACGH] E. Arbarello, M. Cornalba, P. A. Griffiths and J. Harris, Geometry of algebraic curves, vol. I (Springer, New York, 1985).

[Br] R. Bryant, 'Conformal and minimal immersions of compact surfaces into the 4-spheres', $J$. Differential Geom. 17 (1982), 455-473.

[BR] F. E. Burstall and J. H. Rawnsley, Twistor theory for Riemannian symmetric spaces, Lect. Notes Math. 1424 (Springer, Berlin, 1990).

[C] E. Calabi, 'Quelques applications de l'analyse complexe aux surfaces d'aire minima', in: Topics in complex manifolds (ed. H. Rossi) (Les Presses de l'Univ. de Montréal, Montréal, 1967) pp. 59-81.

[ES] J. Eells and S. Salamon, 'Twistorial construction of harmonic maps of surfaces into fourmanifolds', Ann. Scuola Norm. Sup. Pisa Cl. Sci. (4) 12 (1985), 589-640.

[F] W. Fulton, Intersection theory (Springer, New York, 1984).

[H] R. Hartshorne, Algebraic geometry (Springer, New York, 1977).

[KL] P. Z. Kobak and B. Loo, 'Moduli of quaternionic superminimal immersions of 2-spheres into quaternionic projective spaces', Ann. Global Anal. Geom., to appear.

[La] H. B. Lawson, 'Surfaces minimales et la construction de Calabi-Penrose', (Séminaire Bourbaki, Exposé 624, 1984), Astérisque 121-122 (1985), 197-211.

[L] B. Loo, 'The space of harmonic maps of $S^{2}$ into $S^{4}$, Trans. Amer. Math. Soc. 313 (1989), 81-102.

[LV1] B. Loo and I. Vainsencher, 'Limits of graphs', Mat. Contemp. 6 (1994), 41-59.

[LV2] _ _Limits of graphs II', Technical report, in preparation.

[W1] J. Wahl, 'The Jacobian algebra of a graded Gorenstein singularity', Duke Math. J. 55 (1987), 843-871.

[W2] _ 'Gaussian maps on algebraic curves', J. Differential Geom. 32 (1990), 77-98.

Department of Mathematics

Lower Kent Ridge Road

National University of Singapore

Singapore 119260

e-mail: bloo@math.nus.edu.sg 